\title{
PASTING AND MODULAR LATTICES
}

\author{
E. FRIED AND G. GRÄTZER
}

(Communicated by Louis J. Ratliff, Jr.)

\begin{abstract}
A classical lattice construction of R. P. Dilworth is the gluing of two lattices. A number of recent papers by A. Slavik, A. Day, and J. Ježek investigated a generalization: pasting. In this note we prove that by pasting two finite modular lattices, one obtains a modular lattice.
\end{abstract}

A classical problem of lattice theory (see, e.g., [3, Problems V.10 and V.11]) has recently been solved: Only three lattice varieties have the Amalgamation Property, namely, $\mathbf{T}$, the trivial variety, $\mathbf{D}$, the variety of distributive lattices, and $\mathbf{L}$, the variety of all lattices. This result was proved in two steps. First, G. Grätzer, B. Jónsson, and H. Lakser [4] proved that $\mathbf{T}$ and $\mathbf{D}$ are the only modular lattice varieties that have the Amalgamation Property. Then A. Day and J. Ježek [1] proved that $\mathbf{L}$ is the only nonmodular lattice variety with the Amalgamation Property.

The method of G. Grätzer, B. Jónsson, and H. Lakser [4] is projective geometric: in a modular lattice variety $\mathbf{V}$ having the Amalgamation Property, a lattice $L$ is amalgamated many times with $M_{3}$, the five element modular, nondistributive lattice, obtaining a complemented modular lattice. Then Frink's Theorem [2] gives an embedding of $L$ into a projective geometry.

The method of A. Day and J. Ježek [1] uses a version of amalgamation discussed in A. Slavík [8] (see also G. Grätzer [4, Exercise 12 of Section V.4]); in this paper it will be called pasting (see below for the definition). Pasting is stronger than gluing but much weaker then amalgamation. They prove the following theorem: Let $\mathbf{V}$ be a nonmodular variety of lattices; if $\mathbf{V}$ is closed under the pasting of finite lattices, then $\mathbf{V}=\mathbf{L}$.

A. Day and J. Ježek raise the following problem: Can one prove the result of G. Grätzer, B. Jónsson, and H. Lakser on the nonexistence of modular nondistributive lattice varieties having the amalgamation property using this method. More specifically: can one start with $M_{3}$, and obtain $N_{5}$ (the five element

Received by the editors October 30, 1987.

1980 Mathematics Subject Classification (1985 Revision). Primary 06B05, 06C05; Secondary $08 \mathrm{~B} 25$.

Key words and phrases. Pasting, modular lattices, Amalgamation Property, variety.

The research of both authors was supported by the NSERC of Canada. 
nonmodular lattice) by pasting and by forming sublattices and homomorphic images?

In this note we answer this question in the negative. Namely, we prove the following

Theorem. The variety $\mathbf{M}$ of all modular lattices is closed under the pasting of finite lattices.

First, the definition of pasting: Let $L$ be a lattice. Let $A, B$, and $S$ be sublattices of $L, A \cap B=S, A \cup B=L$. Then $L$ pastes $A$ and $B$ together over $S$, if every amalgamation of $A$ and $B$ over $S$ contains $L$ as a sublattice. More precisely:

Definition. Let $L$ be a lattice. Let $A, B$, and $S$ be sublattices of $L, A \cap B=S$, $A \cup B=L$. Let $f_{A}$ and $f_{B}$ be the embeddings of $A$ and $B$, respectively, into $L$. $L$ pastes $A$ and $B$ together over $S$ if whenever $g_{A}$ and $g_{B}$ are embeddings of $A$ and $B$ into a lattice $K$ satisfying $x g_{A}=x g_{B}$ for all $x \in S$, then there is an embedding $h$ of $L$ into $K$ satisfying $f_{A} h=g_{A}$ and $f_{B} h=g_{B}$ (see Figure 1).

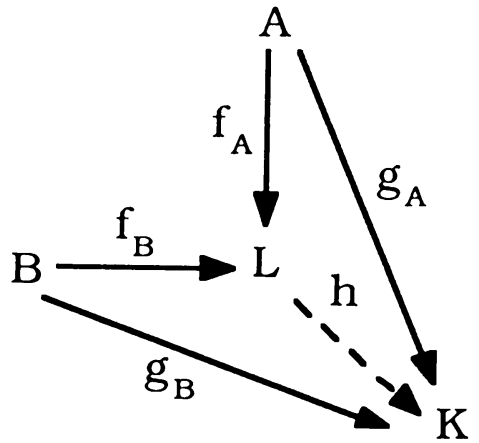

FIGURE 1

A. Day and J. Ježek [1] sharpen a result of A. Slavik [8] with the following characterization of pasting in the finite case.

Proposition. Let $L$ be a finite lattice. Let $A, B$, and $S$ be sublattices of $L$, $A \cap B=S, A \cup B=L . L$ pastes $A$ and $B$ together over $S$ if and only if the following two conditions hold:

(1) For $a \in A$ and $b \in B$, if $a<b$, then there exists an $s \in S$ satisfying $a \leq s \leq b ;$ and dually.

(2) For $s \in S$, all the covers of $s$ in $L$ are in $A$ or all are in $B$; and dually.

This Proposition has the following: 
Corollary. Let $L$ be a finite lattice that pastes $A$ and $B$ over $S$. Let $u, v \in L$ be choosen in such a way that $S \cap[u, v]$ is not empty. Define $L_{1}=[u, v]$, $A_{1}=A \cap[u, v], B_{1}=B \cap[u, v], S_{1}=S \cap[u, v]$. Then $L_{1}$ pastes $A_{1}$ and $B_{1}$ over $S_{1}$.

Proof. It is obvious that if $L, A, B$, and $S$ satisfy (1) and (2), then so do $L_{1}, A_{1}, B_{1}$, and $S_{1}$.

For elements $a, b, c$ of the lattice $L$, we shall use the notation $N_{5}(a, b, c)$ to indicate that $a, b, c$ generate a sublattice isomorphic to $N_{5}$; more precisely, $N_{5}(a, b, c)$ means that $a<b$, both $a$ and $b$ are incomparable with $c, a \wedge c=$ $b \wedge c$, and $a \vee c=b \vee c$.

Now we start proving the Theorem. By way of contradiction, let $L$ be a finite nonmodular lattice, and let $L$ be the pasting of the modular sublattices $A$ and $B$ over $S$. We can choose $L$ as a minimal such lattice.

Since $L$ is nonmodular, it contains elements $a, b, c$ satisfying the condition $N_{5}(a, b, c)$. Clearly, $a \wedge c=0$ and $b \vee c=1$; indeed if $a \wedge c=u$ and $b \vee c=v$, and $0<u$ or $v<1$, then applying the Corollary to $u$ and $v$ we get a smaller lattice, contradicting the minimality of $L$. Thus $L$ is almost modular: every proper interval of $L$ is modular.

We can further assume that in $L \quad a$ is the smallest element $x$ satisfying $N_{5}(x, b, c)$, that is, if $x<a$, then $N_{5}(x, b, c)$ fails to hold. Dually, we can assume that $b$ is a maximal element for which $N_{5}(a, b, c)$ holds.

Claim. $a \succ 0$ and $1 \succ b$.

Proof. Let $0<x<a$ in $L$. First, we verify that $x=b \wedge(x \vee c)$. Indeed, if $x<b \wedge(x \vee c)$, then $N_{5}(x, b \wedge(x \vee c), c)$. Now $x \vee c<1$, since $x \vee c=1$ would contradict the minimality of $a$. Thus $N_{5}(x, b \wedge(x \vee c), c)$ gives us a proper sublattice isomorphic to $N_{5}$, contradicting the minimality of $L$. This shows that $x=b \wedge(x \vee c)$. This however implies that $N_{5}(a, b, x \vee c)$, yielding an $N_{5}$ with $x$ as the zero, contradicting the minimality of $L$. This proves the first part of the claim; the second part follows by duality.

So we are given $a, b, c$ satisfying $N_{5}(a, b, c), a \succ 0$ and $1 \succ b$. We choose $u$ in $[0, c]$ so that $u \succ 0$; we choose $v$ in $[c, 1]$ so that $1 \succ v$. Now we distinguish two cases.

Case 1. $a \vee u=1$ and $b \wedge v=0$.

Case 2. $a \vee u<1$ (or dually).

If Case 1 holds (see Figure 2), then by condition (2) of the Proposition, $a$, $u \in A$ or $B$ and $b, v \in A$ or $B$. We cannot have $a, u \in A$ and $b, v \in A$, because then $N_{5}(a, b, u)$ in $A$, contradicting the modularity of $A$. Similarly for $B$. Hence, by symmetry of $A$ and $B$, we can assume that $a, u \in A$ and $b, v \in B$. This implies that $0,1 \in S$.

By condition (1) of the Proposition, we can choose $t \in[a, b]$ and $w \in[u, v]$, $t, w \in S$. Observe that $t \wedge w=0$ and $t \vee w=1$. Since $a<t$ or $t<b$, by 


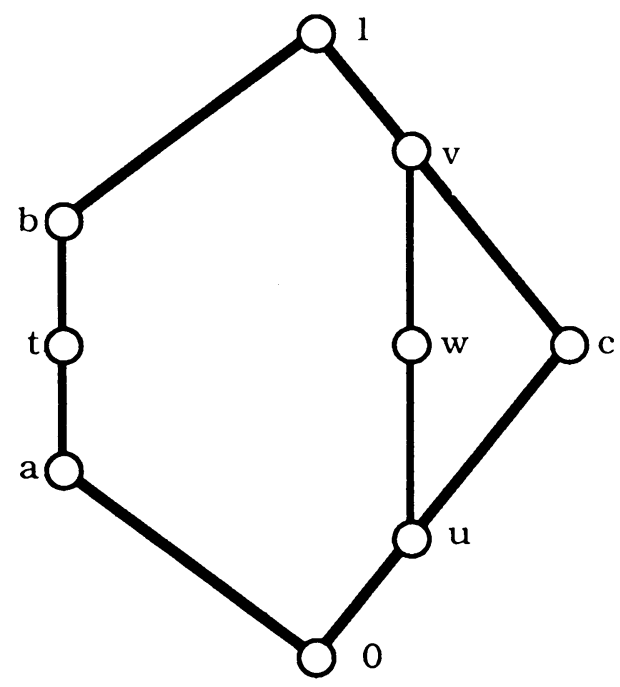

FIGURE 2

reason of symmetry we can asssume that $a<t$. Now $N_{5}(a, t, w)$ and all three elements are in $A$, contradicting the modularity of $A$.

If Case 2 holds (see Figure 3), then $a \vee u$ covers both $a$ and $u$ since [0, $a \vee u$ ] is modular. Since $u<c$, we can choose $x$ in $[u, c], x>u$. We now verify that $N_{5}(a, b \wedge(a \vee x), x)$. Observe that $[u, c]$ and $[a \vee u, 1]$ are perspective intervals $(a \vee u \vee c=1$ is obvious and $(a \vee u) \wedge c=u$ follows from the fact that $a \vee u \succ u$ ), and $[a \vee u, 1]$ and $[a, b]$ are perspective intervals (use that $1 \succ b)$, so $a \vee x \succ a \vee u$, and $b \wedge(a \vee x) \succ a$ by the modularity of $[u, 1]$ and $[a, 1]$. In particular, $a<b \wedge(u \vee x)$. It is obvious that $x \wedge a=x \wedge(b \wedge(a \vee x))$ and $x \vee a=x \vee(b \wedge(a \vee x))$, verifying that $N_{5}(a, b \wedge(a \vee x), x)$.

$N_{5}(a, b \wedge(a \vee x), x)$ means that $L$ has a sublattice isomorphic to $N_{5}$ in $[0, a \vee x]$. By the minimality of $L$, we must then have $a \vee x=1$. Since $a \vee u \succ u, 1 \succ x$, therefore $x=c$. So we get the lattice of Figure 3 as a cover preserving sublattice of $L$.

Now apply the dual of condition (2) to the element 1 ; we obtain that $b$, $c, a \vee u$ are in $A$ or $B$, say in $A$. Since these three elements generate the whole lattice, we get that $A$ is nonmodular, a contradiction. This contradiction proves the Theorem.

In conclusion, we mention a few problems.

Problem 1. Does the Theorem hold for infinite lattices?

Problem 2. In [5], continuumly many modular lattice varieties are given that are closed under gluing. Are they also closed under finite pasting? 


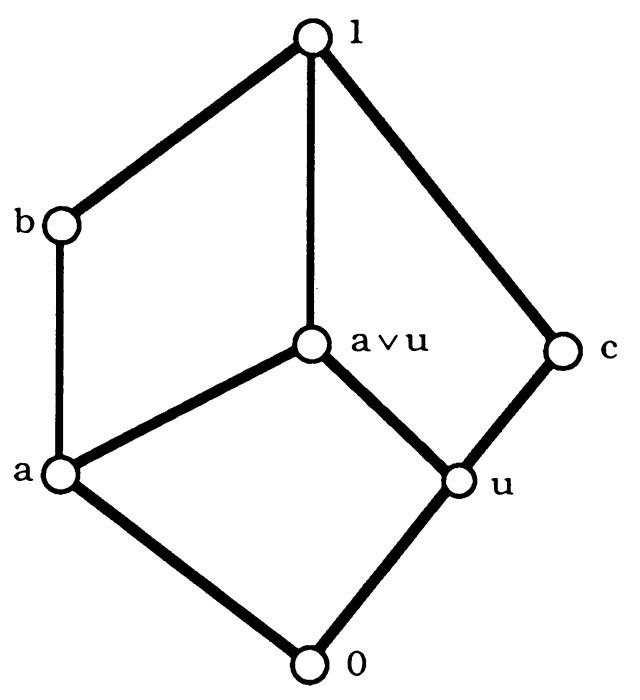

FIGURE 3

Problem 3. There are two "extremal" cases of pasting: gluing and the insertion of a lattice in the critical interval of $N_{5}$. Can all pastings be "put together" from these two special cases?

If a lattice variety $\mathbf{V}$ is closed under gluing, then by $G$. Grätzer and $D$. Kelly [5], $\mathbf{V} \circ \mathbf{D}$ (the variety product of $\mathbf{V}$ and $\mathbf{D}$ ) is a variety again. By a result of $\mathbf{R}$. McKenzie and T. Harrison (see T. Harrison [6]), if $\mathbf{V}$ is a nonmodular lattice variety and $\mathbf{V} \circ \mathbf{D}$ is a variety, then $\mathbf{V}=\mathbf{L}$. These two results combine: If $\mathbf{V}$ is a nonmodular variety closed under gluing, then $\mathbf{V}=\mathbf{L}$. This is a sharpening of the Day-Ježek result. The question is whether the finitary version is true.

Problem 4. If $\mathbf{V}$ is a nonmodular lattice variety and $\mathbf{V}$ is closed under the gluing of finite lattices, is $\mathbf{V}=\mathbf{L}$ ?

\section{REFERENCES}

1. A. Day and J. Ježek, The amalgamation property for varieties of lattices, Mathematics Report, Lakehead University 1 (1983).

2. O. Frink, Complemented modular lattices and projective spaces of infinite dimension, Trans. Amer. Math. Soc. 60 (1946), 452-467.

3. G. Grätzer, General Lattice Theory, Academic Press, Pure and Appl. Math. series, New York, N.Y., 1978.; Birkhäuser Verlag, Matematische Reihe, Band 52, Basel, 1978.

4. G. Grätzer, B. Jónsson, and H. Lakser, The Amalgamation Property in equational classes of modular lattices, Pacific J. Math. 45 (1973), 507-524.

5. G. Grätzer and D. Kelly, Products of lattice varieties, Algebra Universalis 21 (1985), 33-45.

6. $T$. Harrison, $P$ a nontrivial modular lattice variety and $\mathrm{V}$ a nonmodular lattice variety such that $\mathbf{V} \circ \mathbf{P}$ is a variety implies $\mathbf{V}$ is the variety of all lattices, $\mathbf{P h} . \mathrm{D}$. Thesis, University of Hawaii, 1985. 
7. J. Ježek and A. Slavík, Primitive lattices, Czech. Math. J. 29 (104) (1979), 595-634.

8. A. Slavik, $A$ note on the amalgamation property in lattice varieties, Comm. Math. Univ. Carolinae 21 (1980), 473-478.

Department of Mathematics, University of Manitoba, Winnipeg, Manitoba R3T 2N2 CANADA 